\title{
Polymyositis after propylthiouracil treatment for hyperthyroidism
}

\author{
WILLIAM J SHERGY AND DAVID S CALDWELL \\ From the Duke University Medical Center, Durham, North Carolina 27710, USA
}

SUMMARY An additional disorder in the spectrum of thyroid related muscle disease is presented. $\begin{gathered}\omega \\ \Delta\end{gathered}$ Hypothyroid and hyperthyroid disease are both associated with a variety of muscle abnormali- 은 ties, from myalgias to myopathy. Polymyositis, however, has never been reported immediately $\rightarrow$ after treatment for active hyperthyroidism. A patient is presented with typical hyperthyroidism, $\frac{7}{0}$ who developed a severe proximal muscle weakness and a raised creatine phosphokinase after treatment for hyperthyroidism with propylthiouracil $(100 \mathrm{mg}$ orally, three times a day). $\vec{\oplus}$ Electromyography, muscle biopsy, and the course of the patient's illness were consistent with $\$$ polymyositis. Whether this represents a cause-effect association or a chance occurrence is unknown. Physician awareness of the occurrence of a variety of muscle disorders including polymyositis in thyroid disease is emphasised. A brief discussion of thyroid myopathy, thionamide drug reactions, and polymyositis is included.

Key words: myositis, drug reaction, thyroid disease.

A variety of musculoskeletal manifestations associated with thyroid disease may mimic other rheumatic disorders such as polymyalgia rheumatica, periarthritis, periostitis, adhesive capsulitis, fibromyalgia, and rheumatoid arthritis. ${ }^{1-4}$ The most common rheumatic manifestation of thyroid disease is muscle dysfunction. ${ }^{56}$ Hyperthyroidism has been associated with a myopathy, exophthalmia, ophthalmoplegia, periodic paralysis, myasthenia gravis, and a single report of rhabdomyolysis. ${ }^{47}$ Hypothyroidism has been associated with muscle stiffness, myalgias, myxoedema, and a syndrome very similar to polymyositis. ${ }^{8}$ Treatment of hyperthyroidism with thionamides has also been recognised as a cause of a syndrome characterised by arthralgias and myalgias, as well as a 'lupus-like' syndrome. ${ }^{\text {'-12 }}$ Previously, hyperthyroidism and its treatment have never been associated with polymyositis. The patient presented here shows a disorder indistinguishable from

Accepted for publication 6 October 1987.

Correspondence to Dr David S Caldwell. PO Box 2978. Duke University Medical Center. Durham. NC 27710. USA. idiopathic polymyositis after brief treatment with propylthiouracil for hyperthyroidism.

\section{Case report}

A 35 year old white woman had a one year history of increasing tremors, sweats, anxiety, and palpitations associated with a thyroxine (T4) of $258.7 \mathrm{nmol} / \mathrm{l}$ (normal range (NR) $70 \cdot 8-148 \cdot 0$ ), a tri-iodothyronine (T3) uptake of 0.4 (NR $0.35-0.45)$, and a free thyroxine index of 8 (NR $2 \cdot 2-4 \cdot 7)$. A thyroid $N$ scan showed a diffuse homogeneous uptake. She received propylthiouracil $100 \mathrm{mg}$ orally three times a day and noted improvement in her symptoms after $\omega$ two weeks of treatment. She developed, however, myalgias, arthralgias, fever, maculopapular rash, and abnormal liver function tests (serum alanine $\mathbb{D}$ transaminase (serum ALT) $73 \mathrm{U} / 1$, serum aspartate transaminase (serum AST) $97 \mathrm{U} / \mathrm{l}$, alkaline phosphatase $184 \mathrm{U} / \mathrm{l})$, and an increased creatine phosphokinase (CPK). Propylthiouracil was discontinued, prednisone $20 \mathrm{mg}$ daily started, and $\stackrel{\mathbb{Q}}{\Omega}$ $237 \mathrm{MBq}$ of ${ }^{131} \mathrm{I}$ administered. Within four days 2 the arthralgias, rash, and fever had resolved; the 
patient, however, began to experience weakness, and within one week was unable to comb her hair and arise from a seated position. CPK determination was $10380 \mathrm{IU} / \mathrm{l}$. Prednisone was increased to $40 \mathrm{mg}$ daily, and the patient was transferred to Duke University Medical Center for further evaluation and treatment.

On arrival she complained only of weakness. Physical examination showed a thin, white woman with irregular pulse of 86 beats a minute, temperature of $36 \cdot 8^{\circ} \mathrm{C}$, and a mildly enlarged, smooth, nontender thyroid gland. Muscles were non-tender and strength testing showed $5 / 5$ strength distally with $4 / 5$ strength of the quadriceps, biceps, and triceps, $3 / 5$ strength of the deltoid, neck flexors, and extensors, and $2 / 5$ strength of the hip musculature. The patient was unable to stand from a squat or to keep her arms above her head. No rash, lymphadenopathy, synovitis, or muscle atrophy was noted and a neurological examination was normal. Laboratory studies included haemoglobin $121 \mathrm{~g} / \mathrm{l}$, packed cell volume 0.37 , white blood cell count $14.8 \times 10^{9} / 1$ with $85 \%$ segments, $4 \%$ bands, $8 \%$ lymphocytes, and $3 \%$ mononuclear cells. Stool was haemoccult negative and the sedimentation rate was $32 \mathrm{~mm} / \mathrm{h}$. Blood urea was $3.57 \mathrm{mmol} / \mathrm{l}$, creatinine $62 \mu \mathrm{mol} / \mathrm{l}$, and CPK $5205 \mathrm{IU} / \mathrm{l}$. Other enzyme studies showed aldolase $355 \mathrm{U} / \mathrm{l}$, serum ALT $620 \mathrm{U} / \mathrm{l}$, and serum AST $312 \mathrm{U} / \mathrm{l}$. Fluorescent antinuclear antibodies (FANAs) and rheumatoid factor were both negative and whole haemolytic complement was normal. Thyroid function studies were normal with a free $\mathrm{T} 3$ of $5.2 \mathrm{pmol} / \mathrm{l}$, free T4 $15.4 \mathrm{pmol} / \mathrm{l}$, and thyroid stimulating hormone $0 \cdot 16$ arb. unit. Antithyroid antibodies were negative and the chest $x$ ray showed no evidence of active disease.

Corticosteroid treatment was continued with methylprednisolone $10 \mathrm{mg}$ intravenously every six hours. Electromyography of the left deltoid, vastus lateralis, anterior tibialis, and biceps showed profuse fibrillation with decreased duration of motor unit potential, increased complexity, and an increased number of potentials firing for level of effort. A right quadriceps muscle biopsy specimen showed numerous, angulated, atrophic fibres undergoing degeneration and regeneration (Fig. 1). There was only scattered accumulation of lipid droplets. The paucity of inflammatory cells was believed to be secondary to two weeks of corticosteroid treatment before biopsy. Methylprednisolone was consolidated to a single daily dose, and the patient was discharged on prednisone $50 \mathrm{mg}$ daily. At the time of discharge improvement in strength was noted while liver function studies remained only minimally raised, and the CPK had declined to $1570 \mathrm{IU} / \mathrm{l}$.

After doing well for one week, myalgias with

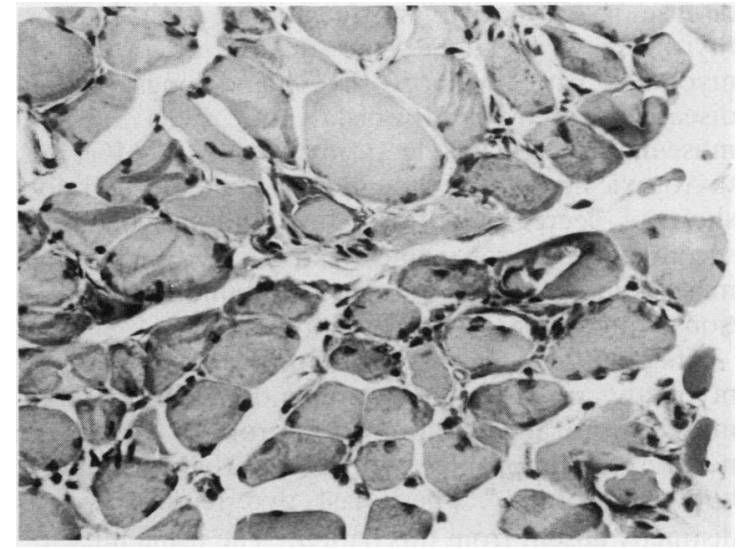

Fig. 1 Right quadriceps muscle biopsy specimen.

weakness recurred following heavy housework. Laboratory studies showed a rise in CPK to 3250 IU/l. Thyroid function studies, liver function tests, and sedimentation rate remained normal. After further parenteral steroid therapy in hospital the patient noted improvement and was discharged on $60 \mathrm{mg}$ prednisone daily. As prednisone was gradually reduced the patient consistently noted recurrence of symptoms with associated rise in CPK when the dosage of prednisone was $30 \mathrm{mg}$ daily. Methotrexate was then introduced and within several weeks the patient began to notice more definite improvement in strength, coincident with a progressive decline to normal of her CPK. The steroid dose was gradually reduced without exacerbation of weakness or rise in CPK.

\section{Discussion}

This patient presented with typical hyperthyroidism and was initially treated with propylthiouracil. This treatment was discontinued because of a drug reaction characterised by myalgias, arthralgias, fever, abnormal liver function tests, and a rash. This syndrome rapidly resolved within four days of prednisone treatment. Proximal muscle weakness developed, however, and progressed to near incapacitation. Electromyography, muscle biopsy, and the patient's course were consistent with polymyositis. Although one may attempt to implicate thyrotoxic myopathy, this is unlikely. The possibility of a myopathy associated with a drug reaction, however. is not so readily dismissed.

Evidence for myopathy can be found in $62-100 \%$ of thyrotoxic patients by electromyography. It will generally resolve with correction of the thyroid 
disease. ${ }^{56}$ Clinically, most patients with hyperthyroidism will have some degree of proximal myopathy with the more severe and longstanding disease also involving distal and even bulbar musculature. ${ }^{56}$ The electromyography (EMG) of thyrotoxic myopathy, though non-specific, generally shows a decrease in fibrillation and fasiculations secondary to a reversible decrease in functioning muscle fibres. ${ }^{56}$ This patient had abundant fibrillations with an increase in potentials.

The muscle biopsy findings in thyrotoxic myopathy are also non-specific and include marked muscle fibre degeneration and atrophy, with connective tissue proliferation and fatty infiltration between fibres. ${ }^{5}$ These last two features were distinctly absent from this patient. The usual muscle enzyme pattern in thyrotoxic myopathy was also absent in this patient. Typically in hyperthyroidism the CPK is normal or decreased. Our patient had dramatically increased CPKs, thus making thyrotoxic myopathy unlikely. Only in the hypothyroid state may the CPK be raised. ${ }^{5}{ }^{1.3}$ In the latter state some authors have advocated the use of CPK as an indicator of response to thyroid replacement treatment. ${ }^{13}$ One report describes hypothyroidism mimicking polymyositis. ${ }^{8}$ Our patient was consistently euthyroid after propylthiouracil treatment when she was most symptomatic from her myopathy and her CPK increases were the greatest.

Propylthiouracil and methimazole are two agents commonly used to treat hyperthyroidism and in general are relatively safe compounds. Several toxic reactions, however, have been reported. ${ }^{14-17}$ Skin eruptions are the most common reaction followed by rheumatic complaints. The latter include myalgias, arthralgias, and arthritis. This constellation of symptoms accompanied by fever and rash has been collectively referred to as the 'antithyroid arthritis syndrome' or 'lupus-like syndrome' or 'collagen-like syndrome' associated with thyroid treatment. ${ }^{1(1-12} 16$

In a discussion of the antithyroid arthritis syndrome Shabtai et al review 500 patients treated with either propylthiouracil or methimazole for hyperthyroidism. "I Eight developed myalgias and polyarthritis, with four of these patients developing fever or rash. Other reports have shown the development of a similar syndrome, often within two weeks of starting treatment with the drug, ${ }^{12} 18$ as in our patient. The patient we report had negative FANAs and a normal complement, whereas several previously reported patients had positive FANAs and decreased complements, ${ }^{10} 18$ implicating an immune mediated disease. Weakness was mentioned but muscle strength was not graded. When measured, CPKs were normal. The drug related syndrome resolved with drug withdrawal, occasionally sup- plemented with a short course of steroids. This patient had resolution of fever, rash, and arthralgias with drug withdrawal and prednisone $20 \mathrm{mg}$ daily; $\stackrel{\mathcal{F}}{\stackrel{9}{+}}$ weakness worsened, however, in association with a marked increase in CPK. This militates against the 'antithyroid arthritis syndrome' accounting for her persistent weakness.

This patient presented with severe proximal muscle weakness, raised CPKs, and an EMG and muscle biopsy suggestive of polymyositis. Although the complete EMG picture of polymyositis generally shows increased insertional activity, the two week course of prednisone before EMG study may have obscured this finding. Also, the paucity of inflammatory cells on the muscle biopsy may likewise be explained.

Idiopathic polymyositis constitutes the largest group of the dermatomyositis-polymyositis complex described by Bohan and Peter. " Typically, pure polymyositis affects more women than men in a ratio of about $2: 1$ and the mean age of presentation is $50 .{ }^{20}$ Most complain of proximal muscle weakness, with $50 \%$ reporting muscle pain. Inability to arise from a seated or squatting position is a frequent presenting sign. CPK is raised in virtually all cases, whereas the sedimentation rate is raised only in $50 \%$ and the FANA test is sometimes positive. EMG shows numerous fibrillation potentials with evidence for denervation and reinnervation, while the muscle biopsy specimen characteristically shows marked atrophy and a predominantly lymphocytic infiltrate. ${ }^{20}$

The long term prognosis for polymyositis is uncertain, with some groups reporting $40 \%$ five year mortality. ${ }^{21}$ Others have shown much more favourable statistics. ${ }^{22}{ }^{2.3}$ Non-steroidal anti-inflammatory agents play no part in the treatment of polymyositis. Treatment is chiefly with corticosteroids, initially in divided doses, then consolidated into a single daily dose. Ultimately an alternate day regimen may be employed. Approximately $75 \%$ of patients will respond to this regimen, allowing for eventual complete withdrawal of treatment. ${ }^{2.3}$ For recalcitrant $\mathrm{N}$ disease or significant steroid toxicity, immunosuppressive treatment with azathioprine or methotrexate is used with variable success. ${ }^{24-27}$ A multicentred trial of cyclosporin $\mathrm{A}$ in polymyositis resistant to all conventional treatment is presently underway.

The patient presented in this report clearly has clinical and laboratory evidence for polymyositis. Although non-diagnostic, the biopsy also supports the diagnosis. The partial response to steroids and flare when these were gradually reduced is typical of many patients with aggressive disease as is the favourable response to the addition of immuno- 
suppressive therapy. An additional interesting feature in this patient is the rapid resolution of the patient's hyperthyroidism with a comparatively short course of propylthiouracil and a low dose of ${ }^{131} I$. The patient has remained euthyroid throughout her course.

Whether this patient represents a new association of two autoimmune diseases, or a unique association of propylthiouracil treatment and polymyositis, or simply the chance occurrence of two diseases is unclear. Certainly the temporal relationship cannot be ignored. A careful search for similar patients may clarify this.

\section{References}

1 Kyle V. Hazleman B L. The thyroid. In: Clinics in rheumatic diseases: Endocrine and metabolic aspects of rheumatic disease. London: Saunders, 1981: 711-22.

2 McGuire J L. Arthropathies associated with endocrine disorders. In: Kelley W N, Harris E D Jr, Ruddy S. Sledgc C B. eds. Textbook of rheumatology, 2nd cd. Philadelphia: Saunders, 1985: 1579-94.

3 Rosenthal M J. Rheumatic manifestations of endocrine discasc. Mediguide to inflammatory diseases. Vol 3. Ncw York: Lawrence Della Corte Publications, 1984: 1-6.

4 Swanson J W, Kelly J J, McCanohey W M. Ncurologic aspects of thyroid dysfunction. Mayo Clin Proc 1981; 56: 504-12.

5 Ramsay I D. Muscle dysfunction in hyperthyroidism. Lancet 1966; ii: 931-4.

6 Ramsay I D. Thyrotoxic muscle discasc. Postgrad Med J 1968: 44: 385-97.

7 Bennet W R, Houston D P. Rhabdomyolysis in thyroid storm. Am J Med 1984; 77: 733-5.

8 Pellman E J, Sumner P, Greenwald A G. Thyroid myopathy mimicking polymyositis. Clinical Rheumatology in Practice 1984: 224-7.

9 Burch W M. Pscudothyrotoxic myopathy: a complication of thionamide therapy in hyperthyroidism. South Med J 1979; 72: 1494-5.
10 Shabtai R. Shapiro M S. Orenstein D. Taragan R. Skenkman L. The antithyroid arthritis syndrome reviewed. Arthritis Rheum 1984; 27: 227-9.

11 Takswa N, Kajimi I, Ogata E. Lupus-like syndrome-a rare complication in thionamide therapy for Grave's discasc. Endocrinol Jpn 1981; 28: 663-7.

12 Hung W. August G P. A 'collagen-like' syndrome associated with antithyroid therapy. J Pediatr 1973; 82: 852-4.

13 Craig R A. Smith J C. Serum creatinc phosphokinase activity in altered thyroid states. Journal of Clinical Endocrinology 1965: 25: 723-31.

14 Barnes H V. Blizzard R M. Antithyroid therapy for toxic diffusc goiter (Grave's discasc): thirty years experience in children and adolescents. J Pediatr 1977: 91: 313-20.

15 Resorc M H. Agranulocytosis and antithyroid drugs. West J Med 1977; 126: 339-43.

16 Amrhein J A. Kenny F M. Ross D. Granulocytopenia, lupuslike syndrome, and other complications of propylthiouracil therapy. J Pediatr 1970; 76: 54-63.

17 Hanson J S. Propylthiouracil and hepatitis. Two cases and a review of the literature. Arch Intern Med 1984: 144: 994-6.

18 Farbman K. Whecler M. Glick S M. Arthritis induced by antithyroid medication. NY State J Med 1969; 69: 826-31.

19 Bohan A. Peter J B. Polymyositis and dermatomyositis. N Engl J Med 1975: 292: 344-50.

20 Bradley W G. Inflammatory discases of muscle. In Kelley W N, Harris E D Jr. Ruddy S. Sledge C B, cds. Textbook of rheumatology, 2nd cd. Philadelphia: Saunders, 1985: 1225-45.

21 Riddoch D. Morgan-Hughes J A. Prognosis in adult polymyositis. J Neurol Sci 1975; 26: 71-5.

22 DeVere R, Bradlcy W A. Polymyositis: its presentation, morbidity and mortality. Brain 1975; 98: 637-41.

23 Bohan A. Peter J B, Bowman R L. Pearson C M. A computer assisted analysis of 153 patients with polymyositis and dermatomyositis. Medicine (Baltimore) 1977: 56: 255-63.

24 Malaviya A N. Many A. Schwartz R S. Treatment of dermatomyositis with methotrexatc. Lancet 1968; ii: 485-7.

25 Bunch T W. Prednisonc and azathioprine for polymyositis: long term follow-up. Arthritis Rheum 1981: 24: 45-51.

26 Arnett F C. Whelton J C. Zizic T. Stevens M B. Methotrexate therapy in polymyositis. Ann Rheum Dis 1973; 32: 536-46.

27 Metzger A L, Bohan A. Goldberg L S, et al. Polymyositis and dermatomyositis: combined methotrexate and corticosteroid therapy. Ann Intern Med 1974; 81: 182-9. 